\section{Panorama de la seguridad y salud en el trabajo de microempresas colombianas ubicadas en un barrio del Municipio de Itagüí, Antioquia, Colombia}

\author{
Workplace safety and health in micro businesses \\ in a neighborhood in the city of Itagüí, \\ Antioquia, Colombia
}
Cenário da segurança e da saúde no trabalho de microempresas colombianas localizadas em um bairro do Município de Itagüí, Antioquia, Colômbia

Jonathan Osorio-Vasco 1

doi: 10.1590/0102-311X00175320

\section{Resumen}

Esta investigación pretendió describir el panorama de la seguridad y salud en el trabajo (SST), con base en los componentes del Sistema de Gestión de Seguridad y Salud en el Trabajo (SGSST), la identificación y control de peligros ocupacionales y la normatividad colombiana, relacionada con el SST de microempresas de los sectores comercial y de servicios del barrio Los $\mathrm{Na}$ ranjos del Municipio de Itagüi, Antioquia, Colombia. La investigación siguió un diseño descriptivo con la participación voluntaria de 50 microempresas. Se utilizó un cuestionario elaborado para la investigación en tres fases: revisión teórica, validación por expertos y pilotaje. En el cuestionario se incluyó: características de la empresa, componentes del SGSST, normatividad en SST $y$ gestión de peligros que incluyó la identificación y control de los peligros. Se encontró que el $88 \%$ de las microempresas no asignan a una persona que maneje el SGSST y el 72\% no afilia de manera permanente los trabajadores al Sistema General de Seguridad Social Integral. Los peligros físicos y químicos no se identifican y controlan de manera constante en el 34\% y 38\% de las microempresas respectivamente. El 93,86\% promedio de las microempresas no conocen la normatividad colombiana relacionada con la SST. El panorama de la SST en un pequeño sector de negocios comerciales y de servicios, no es alentador frente a la adecuación del SGSST, la gestión de peligros y la adecuación e implementación de normas colombianas relacionadas con SST, obligatorias para el cuidado de los trabajadores.

Salud Laboral; Riesgos Laborales; Gestión de Riesgos; OHSAS 18000

\author{
Correspondencia \\ J. Osorio-Vasco \\ Corporación Universitaria Minuto de Dios-UNIMINUTO. \\ Carrera 45\#22D-25, Bello, Antioquia 051051, Colombia. \\ jvascoosori@uniminuto.edu.co \\ 1 Corporación Universitaria Minuto de Dios-UNIMINUTO, \\ Bello, Colombia.
}




\section{Introducción}

Desde sus inicios, la Organización Internacional del Trabajo (OIT) ha promulgado diferentes conceptos sobre el cuidado de la salud laboral de los trabajadores, no obstante, solo desde la década de los 1980's del siglo XX hasta la actualidad tiene reconocidos para todos sus países miembros 40 instrumentos para la seguridad y salud en el trabajo (SST) 1.

Por su parte, la Comunidad Andina de Naciones (CAN), integrada por los países suramericanos: Bolivia, Colombia, Ecuador y Perú, acordó a través de la Decisión no 584 de 2004 un instrumento de SST, el cual orienta la adecuación de políticas públicas integradas por el Estado, los empleadores y los trabajadores en procura del cuidado del bienestar físico, mental y social en el trabajo 2.

Por medio de la Ley no 1.562 de 2012, Colombia incorporó un instrumento de SST que modificó la denominación de Sistema General de Riesgos Profesionales (SGRP) (creado por el Decreto Ley no 1.295 de 1994), por Sistema General de Riesgos Laborales (SGRL), incluyendo la creación del Sistema de Gestión de Seguridad y Salud en el Trabajo (SGSST) 3,4. De igual forma, la Ley no 1.562 de 2012, constituye un antecedente para el Plan Decenal de Salud Pública (PDSP) 2012-2021 con la dimensión 8: Salud y Trabajo, que incluye dos componentes: SST y situaciones prevalentes de origen laboral 5. Tanto la Ley no 1.562 de 2012 y el PDSP son a su vez antecedentes del Plan Nacional de Seguridad y Salud en el Trabajo 2013-2021 que contiene la estrategia para el cumplimiento de las metas incluidas en la dimensión Salud y Trabajo del PDSP 6.

A pesar de las actuales apuestas por la SST, en Colombia existían normas anteriores a la Ley no 1.562 de 2012 que obligaban a las empresas a proteger la salud y la seguridad de los trabajadores, las cuales se remontan a la configuración del sistema de salud durante la mitad del siglo XX, donde se incorporó la atención en salud a los trabajadores a través del Instituto Colombiano de Seguro Social 7. La primera norma que incluyó un capítulo relacionado con la Salud Ocupacional por parte del Ministerio de Salud (hoy denominada por la Ley no 1.562 de 2012 como SST) fue la Ley no 9 de 1979, nombrada como Ley Sanitaria y extendió las garantías de protección ocupacional de los trabajadores colombianos 7. Por otra parte, el Ministerio del Trabajo y Seguridad Social expide la Resolución no 2.400 de 1979, con la estructura del reglamento de seguridad industrial, entregando contenido normativo y técnico sobre la adecuación de las estaciones de trabajo para las empresas 7,8. Las anteriores normas aún están vigentes.

La Resolución no 2.013 de 1986 crea los Comité Paritarios de Salud Ocupacional (hoy denominado Comité Paritarios de Seguridad y Salud en el Trabajo) y crea los programas de: medicina preventiva e higiene y seguridad industrial 9. Posteriormente, la Resolución no 1.016 de 1989 obligó a las empresas a elaborar un Programa de Salud Ocupacional (PSO) (actualmente denominado SGSST por la Ley no 1.562 de 2012), con los lineamientos para el funcionamiento de los programas de manera estructurada para la ejecución del PSO 10.

Actualmente, el SGSST está reglamentado en el capítulo 6 del Decreto Ley no 1.072 de 2015, nombrado como el Decreto Único del Sector Trabajo 11, y se ha convertido en uno de los principales instrumentos (junto con el Código Sustantivo del Trabajo) de soporte y apoyo que deben cumplir todas las empresas en el país, porque incluye la compilación de leyes, decretos, resoluciones y políticas públicas para el cuidado de los trabajadores.

Para el cumplimiento del SGSST, la Resolución no 312 de 2019 estructuró estándares mínimos de implementación según el nivel de riesgo y el número de trabajadores de la empresa. La cantidad de estándares mínimos de cumplimiento está dividida en tres partes: (1) empresas entre los niveles de riesgo I a III y con menos de 10 trabajadores: 7 estándares; (2) empresas entre los niveles de riesgo I a III y entre 11 y 50 trabajadores: 22 estándares y (3) empresas entre los niveles de riesgo IV y V sin importar el número de trabajadores: 62 estándares 12.

Las empresas en Colombia se clasifican bajo dos condiciones, según el Decreto no 957 de 2019, la primera como micro, pequeñas, medianas y grandes empresas según el número de ingresos por las actividades ordinarias anuales y la segunda por el sector económico en el cual se desarrolla la actividad económica: comercio, servicios o manufacura ${ }^{13}$. El cumplimiento de los estándares mínimos para la adecuación del SGSST usualmente está relacionado con el tamaño de la empresa, a pesar que la distribución de cumplimiento de los estándares mínimos no tiene una relación directa con el tamaño de las empresas. 
Las microempresas fueron el 96,4\% de las empresas a 2010 en Colombia, siendo el mayor porcentaje según el Departamento Administrativo Nacional de Estadística (DANE) 14. Sin embargo, son las que obtienen menos ingresos anuales, en consecuencia ha habido poco avance en inversión e implementación 15 para el cumplimiento del SGSST y la normatividad vigente en SST, a pesar que los estándares mínimos se diseñaron con la intención de no hacer grandes inversiones en la adecuación e implementación de los SGSST por parte de las microempresas 16.

El SGSST cobra mayor importancia cuando se analizan cifras de accidentalidad y enfermedad laboral, como las reportadas por la II Encuesta Nacional de Condiciones de Seguridad y Salud en el Trabajo en el Sistema General de Riesgos (II ENCSST), publicada en el año 2014, la cual recogió los datos de 10 aseguradoras de riesgos laborales en Colombia ${ }^{17}$. Esta encuesta reportó que las lesiones musculoesqueléticas son la mayor tendencia de enfermedad laboral en Colombia durante los años 2009 a 2012, siendo el año 2012 con la mayor tendencia (90\%). Respecto a la accidentalidad laboral, la II ENCSST informó que hubo un aumento de 1,41 puntos porcentuales frente la primer encuesta nacional 17. El Departamento de Antioquia reportó una accidentalidad de 19,5\% respecto al total nacional y el 93,3\% de los accidentes son propios del trabajo 17.

El Municipio de Itagüí está ubicado al sur de Medellín (Departamento de Antioquia - Colombia), hacia el año 2012 el 91.2\% de las empresas registradas en el municipio eran microempresas 18. Según estadísticas de la Federación de Aseguradores Colombianos (FASECOLDA), en el año 2019 ocurrieron 8.982 accidentes de trabajo calificados en el Municipio de Itagüí, donde el 11,8\% de los accidentes de trabajo calificados correspondieron al sector comercial de los niveles de riesgo y I, II y III 19. Entre los años 2009 y 2019, se registraron en Itagüí 90.322 accidentes de trabajo calificados en todos los niveles de riesgo. El sector comercial de los niveles de riesgo I, II y III ocurrieron 10.334 accidentes de trabajo calificados, lo que equivale al 11,44\% de los accidentes de trabajo calificados reportados por las empresas entre estos años 19.

Partiendo de lo anterior, la adecuación del SGSST (anteriormente PSO) y las políticas en SST pueden ser muy poco conocidas e interiorizadas para su cumplimiento y aplicación en las microempresas o en las empresas de niveles de riesgo I, II y III con menos de 10 trabajadores del Municipio de Itagüí, puesto que el SGSST es un instrumento que permite la reducción de los accidentes de trabajo calificados. El desconocimiento, y la falta de interés para todo lo relacionado con la SST, pueden ser algunas de las razones por la que aún se presentan accidentes de trabajo calificados en las empresas.

Esta investigación tuvo como objetivo describir el panorama de la SST, con base en los componentes del SGSST, la identificación y control de peligros ocupacionales y la normatividad relacionada con la SST de microempresas en los sectores comercial y de servicios de un barrio ubicado en el Municipio de Itagüí.

\section{Materiales y métodos}

Se realizó un estudio descriptivo con la participación voluntaria de 50 microempresas del barrio Los Naranjos, ubicado en el Municipio de Itagüí. Se invitaron a participar a todas las microempresas (o negocios) ubicados dentro de los límites del barrio, extendiendo la invitación de participar a 80 microempresas (o negocios). Las microempresas cumplieron al menos uno de los siguientes criterios de inclusión: que fueran del sector comercial o de servicios, el límite de ingresos anuales para ser microempresas en Colombia o tener menos de 10 trabajadores y que su clasificación de nivel de riesgo fuera I, II o III. Se contactó inicialmente a través de una carta de invitación dirigida al representante de SST, en caso que no existiera, se contactó al representante legal o a los encargados de las microempresas que tuvieran las facultades de dar respuesta al cuestionario.

Se utilizó un cuestionario que incluyó preguntas sobre: componentes del SGSST, normatividad en SST e identificación y control de peligros ocupacionales. El cuestionario fue elaborado para la investigación y se emplearon tres fases para su elaboración. Durante la primera fase de elaboración se realizó una revisión teórica sobre la SST y de la normatividad vigente en Colombia relacionada con el ámbito laboral y de la SST. De esta revisión se eligieron los siguientes contenidos para el cuestionario: características de la empresa, componentes del SGSST, normatividad en SST y gestión de peligros con la identificación y control de los peligros ocupacionales. Para cada una de las variables de los 
componentes del SGSST y la gestión de peligros se eligió la siguiente escala de valoración: (1) nunca, (2) muy pocas veces, (3) algunas veces, (4) casi siempre, (5) siempre y para la normatividad en SST se utilizaron las respuestas de "sí" y "no".

La segunda fase de la elaboración fue la validación del cuestionario. El cuestionario fue validado por tres expertos con investigaciones en el campo de la SST, donde dos validadoras fueron internas de la Corporación Universitaria Minuto de Dios-UNIMINUTO - Seccional Antioquia Chocó (Grupo de Investigación ASOCOP) y un validador externo de la Facultad Nacional de Salud Pública de la Universidad de Antioquia (Grupo de Investigación Seguridad y Salud en el Trabajo). La validación se realizó desde dos componentes: el primero validó los componentes técnicos y teóricos de la SST relacionados con el objetivo de la investigación y el segundo evaluó el lenguaje empleado para la comprensión de la población voluntaria a la hora de contestar el cuestionario.

La tercera fase de la construcción del cuestionario fue la aplicación de una prueba piloto para determinar el tiempo promedio de aplicación del cuestionario (20 minutos), hacer el ajuste final del cuestionario y refinar el protocolo de aplicación.

La investigación siguió los lineamientos de la Resolución no 8.430 de 1993 de Colombia, por la cual se establecen las normas científicas, técnicas y administrativas para la investigación en salud. Esta investigación no realizó una intervención sobre la población, de acuerdo con esta resolución y la caracterización planteada en esta, la investigación fue clasificada bajo la categoría de "riesgo mínimo", porque la técnica de recolección de la información se dio a través de un cuestionario. Los microempresarios voluntarios participantes firmaron un consentimiento informado, el cual explicaba los alcances de la investigación y el uso posterior de la información recolectada. Adicionalmente, la investigación contó con el aval ético entregado el 27 de agosto de 2019 por parte del Comité de Ética para la Investigación de la Corporación Universitaria Minuto de Dios-UNIMINUTO .

Se utilizó el software estadístico Stata versión 15 (https://www.stata.com/) para la elaboración de la base de datos que incluyó cada variable del cuestionario. Se utilizó estadística descriptiva con frecuencias en porcentajes para cada una de las variables del estudio.

\section{Resultados}

Los siguientes resultados están agrupados en: características de la empresa, componentes del SGSST, identificación y control de los peligros y normatividad en SST. Se presenta la frecuencia en porcentajes en que se cumple cada una de las variables preguntadas a las personas encargadas de las microempresas que voluntariamente aceptaron resolver el cuestionario.

Las características de las microempresas arrojaron que: el 72\% de las microempresas son del sector comercial y el 28\% de las microempresas formaban parte del sector servicios; el $72 \%$ de las microempresas no tiene o no conoce su nivel de riesgo, mientras que el resto de las microempresas estaban: en el nivel I de riesgo el 20\%, en el nivel II de riesgo el 6\% y en el nivel III de riesgo el 2\%; el $82 \%$ de las microempresas tenía actualizado el registro mercantil, mientras que el 18\% no lo tenía actualizado en el momento de responder el cuestionario; el 92\% de las microempresas que cuentan con una ARL no reciben acompañamiento, capacitación o asesoría por parte de ellas; el 82\% de las microempresas no tiene una contratación definida para sus empleados según los tipos de contratación que existen en Colombia y el 96\% de las microempresas no pertenece a una agremiación de empresas.

En los componentes del SGSST de las microempresas participantes del estudio, se encontró entre los resultados más relevantes que: el 88\% de las microempresas no asignan a una persona que maneje el SGSST de la empresa, el 72\% no afilia de manera permanente los trabajadores al Sistema General de Seguridad Social Integral, el cual incluye la afiliación al SGRL, el 96\% no mantiene de manera continua o no tiene de manera permanente un plan de capacitación para los empleados durante el año, el $80 \%$ no implementa o algunas veces tiene una política de seguridad y salud en el trabajo que se renueve cada año y que se informe a los trabajadores, el $94 \%$ nunca o algunas veces realiza valoraciones médico-ocupacionales de ingreso, seguimiento y egreso y el 72\% nunca, muy pocas o algunas veces se realizan medidas de control preventivo de los peligros identificados (Tabla 1). 
Tabla 1

Componentes del Sistema de Gestión de Seguridad y Salud en el Trabajo (SGSST) preguntados a las microempresas. Municipio de Itagüí, Antioquia, Colombia $(\mathrm{N}=50)$.

\begin{tabular}{|c|c|c|c|c|c|}
\hline Pregunta del cuestionario & $\begin{array}{l}\text { Nunca } \\
\%\end{array}$ & $\begin{array}{l}\text { Muy pocas } \\
\text { veces } \\
\%\end{array}$ & $\begin{array}{l}\text { Algunas } \\
\text { veces } \\
\%\end{array}$ & $\begin{array}{c}\text { Casi } \\
\text { siempre } \\
\%\end{array}$ & $\begin{array}{l}\text { Siempre } \\
\\
\%\end{array}$ \\
\hline $\begin{array}{l}\text { ¿Se identifican las restricciones médico-laborales de los } \\
\text { trabajadores para cada tarea? }\end{array}$ & 64 & 0 & 4 & 18 & 14 \\
\hline $\begin{array}{l}\text { ¿Desarrolla y ejecuta programas de promoción y prevención de } \\
\text { salud laboral? }\end{array}$ & 84 & 0 & 4 & 8 & 4 \\
\hline $\begin{array}{l}\text { ¿La empresa o negocio mantiene la implementación de las } \\
\text { etapas del SGSST según la Resolución no } 312 \text { de 2019? }\end{array}$ & 84 & 0 & 4 & 6 & 6 \\
\hline $\begin{array}{l}\text { ¿La empresa o negocio mantiene actualizada una política de } \\
\text { SGSST y la informa a los trabajadores? }\end{array}$ & 76 & 0 & 4 & 10 & 10 \\
\hline $\begin{array}{l}\text { ¿La empresa o negocio implementa e informa a los } \\
\text { trabajadores de la existencia de un vigía o COPASST? }\end{array}$ & 98 & 0 & 0 & 0 & 2 \\
\hline $\begin{array}{l}\text { ¿La empresa o negocio implementa e informa a los } \\
\text { trabajadores de la existencia de un comité de convivencia? }\end{array}$ & 98 & 0 & 0 & 0 & 2 \\
\hline $\begin{array}{l}\text { ¿Tiene una persona asignada para el manejo del SGSST y } \\
\text { estándares mínimos? }\end{array}$ & 88 & 0 & 0 & 8 & 4 \\
\hline $\begin{array}{l}\text { ¿Los trabajadores se encuentran afiliados al SGRL } \\
\text { permanentemente como también al SGSSI? }\end{array}$ & 70 & 0 & 2 & 4 & 24 \\
\hline $\begin{array}{l}\text { ¿Se asignan recursos de manera directa a las labores } \\
\text { del SGSST? }\end{array}$ & 86 & 0 & 4 & 10 & 0 \\
\hline $\begin{array}{l}\text { ¿Ha elaborado y mantiene actualizado un plan para: } \\
\text { emergencias o ayuda mutua? }\end{array}$ & 86 & 0 & 2 & 8 & 4 \\
\hline $\begin{array}{l}\text { ¿Ha elaborado y ejecuta un plan de capacitación para } \\
\text { el año actual? }\end{array}$ & 88 & 2 & 4 & 4 & 2 \\
\hline $\begin{array}{l}\text { ¿Realiza mantenimiento de los equipos y herramientas } \\
\text { empleados en la empresa de manera periódica (al menos } \\
\text { cada año)? }\end{array}$ & 36 & 0 & 4 & 16 & 44 \\
\hline $\begin{array}{l}\text { ¿Entrega oportunamente equipo de protección personal a los } \\
\text { trabajadores, los capacita y cambia cada vez que se deterioran? }\end{array}$ & 56 & 0 & 2 & 8 & 34 \\
\hline $\begin{array}{l}\text { ¿El pago al SGRL y de la SGSSI que realiza cada mes lo hace a } \\
\text { través de internet u otro medio diferente a ir a un banco? }\end{array}$ & 84 & 0 & 0 & 0 & 16 \\
\hline ¿Aplica la batería de riesgo psicosocial anualmente? & 100 & 0 & 0 & 0 & 0 \\
\hline ¿Desarrolla y ejecuta programas de vigilancia epidemiológica? & 92 & 0 & 4 & 4 & 0 \\
\hline $\begin{array}{l}\text { ¿Tiene las valoraciones médico-ocupacionales de ingreso, } \\
\text { seguimiento y egreso? }\end{array}$ & 90 & 0 & 4 & 0 & 6 \\
\hline $\begin{array}{l}\text { ¿Las condiciones medioambientales del lugar de trabajo } \\
\text { respecto al aire, la temperatura y medio ambiente en general } \\
\text { son óptimas? }\end{array}$ & 60 & 0 & 2 & 10 & 28 \\
\hline $\begin{array}{l}\text { ¿Ha recibido acompañamiento como: capacitación, asesoría y } \\
\text { apoyo por parte de los entes gubernamentales entorno } \\
\text { a la SST? }\end{array}$ & 96 & 2 & 0 & 0 & 2 \\
\hline $\begin{array}{l}\text { ¿Ha recibido o participado en acompañamiento como: } \\
\text { capacitación, asesoría y apoyo por parte de una Cámara de } \\
\text { Comercio u otra agremiación empresarial entorno a la SST? }\end{array}$ & 76 & 2 & 6 & 6 & 10 \\
\hline
\end{tabular}

COPASST: Comité Paritario de Seguridad y Salud en el Trabajo; SGRL: Sistema General de Riesgos Laborales; SGSSI: Sistema de General de Seguridad Social Integral; SST: seguridad y salud en el trabajo.

Fuente: elaboración propia a partir de los resultados del estudio obtenidos de la aplicación del cuestionario. 
Los resultados obtenidos en la gestión de peligros, específicamente en la identificación y control de peligros ocupacionales, se encontró que: el 34\% de las microempresas no identifican y controlan de manera constante los peligros físicos; el 38\% de las microempresas no identifican y controlan de manera constante los peligros químicos; el 44\% de las microempresas no identifican y controlan de manera constante los peligros biológicos; el 30\% de las microempresas no identifican y controlan de manera constante los peligros de condición de seguridad; el 100\% de las microempresas no identifican y controlan de manera constante los peligros psicosociales; y el 38\% de las microempresas no identifican y controlan de manera constante los peligros biomecánicos (Tabla 2).

Frente a la normatividad colombiana relacionada con el SST, el 93,86\% promedio de las microempresas no la conocen; entre las menos conocidas están: la Ley no 1.562 de 2012, el Decreto Ley no 1.072 de 2015 y la Resolución no 312 de 2019 con un 96\% de respuestas en “no” (Tabla 3).

\section{Discusión}

El panorama de la seguridad y salud en el trabajo de un pequeño sector de negocios comerciales y servicios, conformados por microempresas del barrio Los Naranjos, no es alentador frente a la adecuación del SGSST, la gestión de peligros y la adecuación e implementación de normas colombianas relacionadas con SST, obligatorias para el cuidado de los trabajadores.

Los componentes del SGSST que se encuentran en la Resolución no 312 de 2019 como estándares mínimos para las empresas con menos de 10 trabajadores y en los niveles de riesgo I, II y III, junto con otros componentes del sistema de gestión que fueron preguntados en este estudio a las microempresas que participaron, reportaron en un alto porcentaje que "nunca" se implementan y en bajos porcentajes "muy pocas veces" y "algunas veces" mantienen en funcionamiento los componentes del SGSST y siguen las etapas de implementación propuestas en la Resolución no 312 de 2019.

Menos de la mitad de las microempresas participantes del estudio tienen poca o esporádica frecuencia de identificación y control de los peligros: físicos, químicos, biológicos, de condiciones de seguridad y biomecánicos, los cuales forman parte de las tareas iniciales para los SGSST y el cuidado básico de los trabajadores. Sin embargo, los peligros psicosociales no son identificados y evaluados a

Tabla 2

Identificación y control de peligros ocupacionales preguntados a las microempresas. Municipio de Itagüí, Antioquia, Colombia (N = 50).

\begin{tabular}{|c|c|c|c|c|c|}
\hline \multirow[t]{2}{*}{ Pregunta del cuestionario } & Nunca & $\begin{array}{l}\text { Muy pocas } \\
\text { veces }\end{array}$ & $\begin{array}{l}\text { Algunas } \\
\text { veces }\end{array}$ & $\begin{array}{c}\text { Casi } \\
\text { siempre }\end{array}$ & Siempre \\
\hline & $\%$ & $\%$ & $\%$ & $\%$ & $\%$ \\
\hline $\begin{array}{l}\text { ¿Identifica los peligros físicos? (ruido, iluminación, temperatura, vibraciones, } \\
\text { entre otros). }\end{array}$ & 14 & 6 & 14 & 40 & 26 \\
\hline $\begin{array}{l}\text { ¿ldentifica los peligros químicos? (sustancias líquidas, vapores, gases, } \\
\text { entre otros). }\end{array}$ & 22 & 4 & 12 & 38 & 24 \\
\hline ¿Identifica los peligros biológicos? (micro y macro organismos entre otros). & 28 & 2 & 14 & 36 & 20 \\
\hline $\begin{array}{l}\text { ¿ldentifica los peligros de condiciones de seguridad? (mecánicos, } \\
\text { energéticos, seguridad industrial, riesgo público, entre otros). }\end{array}$ & 22 & 0 & 8 & 38 & 32 \\
\hline ¿ldentifica los peligros psicosociales? (batería de riesgo psicosocial) & 100 & 0 & 0 & 0 & 0 \\
\hline $\begin{array}{l}\text { ¿Identifica los peligros biomecánicos? (posturas sedentes, esfuerzo, } \\
\text { movimiento repetitivo, entre otros). }\end{array}$ & 28 & 2 & 8 & 26 & 36 \\
\hline $\begin{array}{l}\text { ¿Realiza medidas de control preventivo sobre los peligros identificados en } \\
\text { las tareas de la empresa? }\end{array}$ & 56 & 6 & 10 & 12 & 16 \\
\hline $\begin{array}{l}\text { ¿ldentifica las condiciones en salud de los trabajadores y la descripción } \\
\text { sociodemográfica? }\end{array}$ & 72 & 4 & 2 & 6 & 16 \\
\hline
\end{tabular}

Fuente: elaboración propia a partir de los resultados del estudio obtenidos de la aplicación del cuestionario. 
Tabla 3

Normatividad colombiana relacionada con la seguridad u salud em el trabajo (SST) preguntada a las microempresas. Municipio de Itagüí, Antioquia, Colombia $(\mathrm{N}=50)$.

\begin{tabular}{|c|c|c|}
\hline Pregunta del cuestionario & Sí & No \\
\hline $\begin{array}{l}\text { ¿Conoce la resolución sobre las actividades de alto riesgo para la salud del trabajador? } \\
\text { (Resolución no } 2.090 \text { de 2003). }\end{array}$ & 10 & 90 \\
\hline ¿Conoce el Estatuto de Higiene y Seguridad Industrial? (Resolución no 2.400 de 1979). & 12 & 88 \\
\hline $\begin{array}{l}\text { ¿Conoce el reglamento de comités de Medicina, Higiene y Seguridad Industrial en lugares de } \\
\text { trabajo? (Resolución no } 2.013 \text { de 1986). }\end{array}$ & 10 & 90 \\
\hline $\begin{array}{l}\text { ¿Conoce la ley que determina la organización y administración del Sistema General de } \\
\text { Riesgos Profesionales? (Ley no } 1.295 \text { de 1994). }\end{array}$ & 6 & 94 \\
\hline $\begin{array}{l}\text { ¿Conoce la ley de fortalecimiento del Sistema General de Seguridad Social en Salud? } \\
\text { (Ley no } 1.438 \text { de 2011). }\end{array}$ & 6 & 94 \\
\hline $\begin{array}{l}\text { ¿Conoce la resolución que establece los parámetros y requisitos para desarrollar, certificar } \\
\text { y registrar la capacitación virtual en el Sistema de Gestión de la Seguridad y Salud en el } \\
\text { trabajo? (Resolución no } 4.927 \text { de 2016). }\end{array}$ & 4 & 96 \\
\hline $\begin{array}{l}\text { ¿Conoce la ley que modifica el sistema de riesgos laborales y se dictan otras disposiciones en } \\
\text { materia de salud ocupacional? (Ley no } 1.562 \text { de 2012). }\end{array}$ & 4 & 96 \\
\hline ¿Conoce el Decreto Único reglamentario del Sector Trabajo? (Decreto no 1.072 de 2015). & 4 & 96 \\
\hline $\begin{array}{l}\text { ¿Conoce la resolución que define los estándares mínimos del Sistema de Gestión de la } \\
\text { Seguridad y Salud en el Trabajo SG-SST? (Resolución no } 312 \text { de 2019). }\end{array}$ & 4 & 96 \\
\hline $\begin{array}{l}\text { ¿Conoce la ley que adopta medidas para prevenir, corregir y sancionar el acoso laboral? } \\
\text { (Ley no } 1.010 \text { de 2006). }\end{array}$ & 4 & 96 \\
\hline $\begin{array}{l}\text { ¿Conoce el Reglamento de Seguridad para protección contra caídas en trabajos con alturas? } \\
\text { (Resolucíon no } 1.409 \text { de 2012). }\end{array}$ & 8 & 92 \\
\hline $\begin{array}{l}\text { ¿Conoce las resoluciones que establecen la conformación y funcionamiento del comité de } \\
\text { Convivencia Laboral en Entidades Públicas y Empresas privadas? (Resolución no } 652 \text { de } 2012 \text { y } \\
\text { (Resolución no } 1.356 \text { de 2012). }\end{array}$ & 4 & 96 \\
\hline $\begin{array}{l}\text { ¿Conoce la resolución por la cual se regula la práctica de evaluaciones médicas-ocupacionales } \\
\text { y el manejo y contenido de las historias clínicas ocupacionales? (Resolución no } 2.346 \text { de 2007). }\end{array}$ & 4 & 96 \\
\hline $\begin{array}{l}\text { ¿Conoce la resolución que establece el control de la exposición a factores de riesgo } \\
\text { psicosocial en el trabajo? (Resolución no } 2.646 \text { de 2008). }\end{array}$ & 6 & 94 \\
\hline Promedio de respuestas & 6,14 & 93,86 \\
\hline
\end{tabular}

Fuente: elaboración propia a partir de los resultados del estudio obtenidos de la aplicación del cuestionario.

través de la batería de riesgo psicosocial del Ministerio del Trabajo. Se resalta que, en la gestión general de los peligros, a excepción de los peligros psicosociales que en un 100\% no se realiza, es común que más del $50 \%$ identifique y controle los peligros: físicos, químicos, biológicos, de condiciones de seguridad y biomecánicos.

En un alto porcentaje, las microempresas no conocen 14 normas que forman parte del marco normativo y jurídico de la seguridad y salud en el trabajo en Colombia. La identificación de normatividad aplicable del SGRL es una etapa fundamental dentro de las labores iniciales para la implementación de los SGSST.

Los estándares mínimos fueron diseñados con la intención de ajustar las necesidades de las microempresas, garantizando el cuidado de la SST con la implementación de niveles mínimos de seguridad en las empresas, y así ajustarse a los costos de cumplimiento en la adecuación de un SGSST 16. Aunque desde el año de 1989 en Colombia las empresas están obligadas a tener un PSO 10, siendo ratificado por la Ley no 1.562 de 2012 que modificó la denominación de PSO por el SGSST y reafirmó la obligatoriedad de su implementación 4, las micro y pequeñas empresas consultadas en este estudio 
no tienen indicios, al parecer, de una implementación clara y concreta sobre el camino a seguir con relación de la SST.

A pesar que la responsabilidad normativa inicialmente recae en el microempresario, no hay que desconocer que las aseguradora de riesgos laborales también tienen responsabilidades. La Ley no 1.562 de 2012 obliga a las aseguradora de riesgos laborales a acompañar y capacitar a los microempresarios para el logro de implementación de cada una de las etapas del SGSST 4. En este estudio se evidencia que las microempresas afiliadas a una aseguradora de riesgos laborales no reciben acompañamiento, capacitación o asesoría. Este resultado también es preocupante, porque las aseguradoras juegan un papel fundamental en el desarrollo del SGSST, pues son las llamadas a dar una correcta orientación a las microempresas en la manera como deben encaminar las acciones de SST y cada uno de los componentes que integra el Sistema de Gestión.

El capítulo 6 del Decreto Ley no 1.072 de 2015, que contiene el bloque normativo de la SGSST, obliga a todas las empresas a identificar peligros y valorar los riesgos ocupacionales, lo cual también fue incluido como obligatorio en los estándares mínimos a través de la Resolución no 312 de 2019 para las empresas con menos de 10 trabajadores o de niveles de riesgo I, II y III, características similares a las microempresas comerciales. A pesar de esta normatividad, aún hay microempresas que desconocen su obligación frente la gestión de peligros ocupacionales al no identificar y controlar los peligros ocupacionales. Lo encontrado en este estudio permite evidenciar que un tercio del porcentaje de las microempresas aún no cumplen con la identificación de peligros y la valoración del riesgo de manera continua, aumentando la probabilidad de ocurrencia de accidentalidad y/o enfermedad laboral. Es de resaltar que un poco más de la mitad de las microempresas de este estudio mantienen la gestión de peligros de manera recurrente al identificar y controlar los peligros diferencia del riesgo psicosocial, con el cual no se hace ningún tipo de gestión por parte de las microempresas participantes del estudio.

Los resultados de este estudio frente al no cumplimiento de la gestión e identificación de los factores de riesgo psicosociales dejan una gran preocupación. Los problemas mentales derivados del estrés laboral en Colombia fueron reportados en la II ENCSST como una de las patologías que tiene mayor reporte a las aseguradora de riesgos laborales y al sistema de salud ${ }^{17}$. La preocupación derivada de estos resultados es mayor cuando en un alto porcentaje también se desconoce el componente normativo que exige la aplicación de la batería de riesgo psicosocial por parte de las empresas.

Mantener una matriz legal actualizada sobre la normatividad en SST en Colombia, y otras adicionales que sean aplicables a la operación de cada empresa, es una de las exigencias documentales requerida y obligatoria que forma parte del SGSST, especificada en el Decreto Ley no 1.072 de 2015, en el artículo 2.2.4.6.12 11. El panorama que entregan los resultados de este estudio muestra que las microempresas no conocen las normas básicas que conforman o conformaban el marco normativo de la SST en Colombia hasta el momento de ejecución de esta investigación.

Los resultados de este estudio, a menor escala, se relacionan con lo reportado por Vega-Monsalve 20 en 2017, quien concluyó sobre lo poco estratégicas que son las empresas frente a la adecuación e implementación del SGSST en empresas con más de 50 empleados en Antioquia. A la luz de estos resultados las políticas en SST no están siendo efectivamente dirigidas hacia las microempresas, porque los resultados de este estudio muestran que en un alto porcentaje las microempresas no recibieron apoyo de ningún ente gubernamental sobre capacitación, asesoría o apoyo sobre el SGSST. Las microempresas que tienen un mínimo nivel de implementación de la SST puede ser solo por el cumplimiento de las normas en SST y no por tener un enfoque de cuidado a la salud de los trabajadores 20.

En este estudio, un poco más de la mitad de las empresas participantes informaron que realizan la gestión de peligros a través de la identificación y valoración de los peligros ocupacionales, exceptuando al riesgo psicosocial. Este registro se basó únicamente en la respuesta del cuestionario facilitado para el estudio y no se verificó que se realizara a través de alguna metodología como la Guía Técnica Colombiana 45 21. En un estudio realizado con empresas colombianas de la ciudad de Bogotá y el Departamento del Valle del Cauca, las microempresas no identificaron factores de riesgo, un porcentaje pequeño los identificó con una metodología y controló los peligros 22. Se destaca entonces que la gestión de peligros de los microempresarios, que en la mayoría de este estudio se preocupan al menos por tener presente la identificación y control de los peligros o factores de riesgo que están presentes en sus actividades y tareas diarias, pero sin tener presentes los riesgos psicosociales que son de gran importancia. 
La aplicación de la normatividad relacionada con la SST en el ámbito empresarial usualmente queda solo sobre el papel 20 y no se le da la suficiente relevancia e importancia que debe tener, especialmente por las sanciones que puede traer el no cumplimiento de la misma. La normatividad para las microempresas desde el año 2017 se ha flexibilizado a través de resoluciones como la Resolución no 1.111 de 2017 y la Resolución no 312 de 2019, las cuales limitaron el número de estándares a cumplir, reduciendo virtualmente el número de normas para su cumplimiento 15. La Resolución no 312 de 2019 obliga a las microempresas cumplir con 7 estándares mínimos de los 62 que conforman el SGSST. Sin embargo, a pesar de estos límites mínimos de cumplimiento, los microempresarios de igual manera deben cumplir con toda la normatividad que está entorno a la SST en Colombia 12,15. Los resultados de este estudio frente al desconocimiento de la normatividad preocupan, pues la no implementación normativa pone en riesgo la seguridad y salud de los trabajadores y las microempresas pueden estar sujetas de sanciones por el incumplimiento.

El estudio contó con la participación voluntaria de 50 microempresas, a pesar que se hizo la invitación a 80 microempresas ubicadas dentro del barrio Los Naranjos. El estudio solo indagó por la gestión de peligros según el conocimiento del encargado de responder el cuestionario, pero no se verificó la aplicación de una metodología y controles a los peligros identificados, al igual que las preguntas sobre la normatividad en SST, que fueron registradas con base en el conocimiento de la persona que respondió el cuestionario (el cual no se verificó ni su formación ni un test previo de conocimientos sobre SST), la cual se esperaba fuera la más idónea por parte de la microempresa sobre los temas de SST, que en su mayoría fueron los dueños o encargados del negocio. Es posible que otras personas o trabajadores diferentes a los dueños o encargados de las microempresas tuvieran más conocimiento sobre el SGSST, la gestión de peligros y normatividad en SST.

Finalmente, el estudio no pretendió señalar directos responsables por el incumplimiento de la SST, sino más bien, pretendió hacer una descripción del panorama actual de la SST en un sector pequeño de microempresas. Posteriores estudios podrán profundizar si el desalentador panorama respecto al SGSST, la gestión de peligros y normatividad en SST es un problema directo de los microempresarios, que no tienen una voluntad de cumplir con la SST, o si está relacionado con la manera como se estructura el SGRL, desde la base de determinantes sociales y económicos en Colombia.

\section{Conclusiones}

El SGSST es uno de los instrumentos más adecuados en la actualidad para cuidar de manera íntegra a los trabajadores, así como también es la herramienta más útil para que cualquier empresa pueda demostrar que realiza una debida identificación y control de los peligros a los cuales expone a los trabajadores en sus tareas diarias 23,24 . Sin embargo, el SGSST aún no impacta de manera directa y positiva en las microempresas que se encuentran entre los niveles de riesgo I, II y III, con menos de 10 empleados de los sectores del comercio y servicios del barrio Los Naranjos.

Las entidades gubernamentales responsables de comunicar las normativas en torno a la SST, y de velar por el cumplimiento de la identificación y control de los peligros ocupacionales para la protección de la salud laboral, deben de plantear estrategias que incluyan a todos los microempresarios. La ausencia de acompañamiento de las entidades gubernamentales con los microempresarios entorno a la SST, evidenciada en este estudio, puede que no sea generalizado, pero sí una posible falta de cobertura en atender a todos los sectores y tipos de empresas, en especial las microempresas que tienen el mayor porcentaje de empleabilidad en Colombia 25.

Otros estudios deberán profundizar en una muestra más amplia y diversa de microempresas, ahondando en sus principales falencias para la implementación del SGSST, la identificación y control de los peligros y el cumplimiento de la normatividad en SST. 


\section{Información adicional}

ORCID: Jonathan Osorio-Vasco (0000-0002-30625736).

\section{Agradecimientos}

Se agradece a las estudiantes del semillero Seguridad y Salud en el Trabajo Aburrá Sur (SSTAS). del Centro Regional Aburrá Sur por su colaboración en el trabajo de campo de este estudio. A la Corporación Universitaria Minuto de Dios-UNIMINUTO y a UNIMINUTO-Seccional Antioquia ChocóCentro Regional Aburrá Sur quienes cofinanciaron este estudio.

\section{Referencias}

1. Organización Internacional del Trabajo. Seguridad y salud en el trabajo: normas del trabajo. https://www.ilo.org/global/topics/safety-andhealth-at-work/lang--es/index.htm (accedido el 11/Oct/2020).

2. Comunidad Andina de Naciones. Decisión no 584. Instrumento Andino de Seguridad y Salud en el Trabajo. http://fondoriesgoslaborales. gov.co/documents/normatividad/decisiones/ Decision-584.pdf (accedido el 11/Oct/2020).

3. Ministerio de Trabajo. Decreto no 1.295. Por el cual se determina la organización y administración del Sistema General de Riesgos Profesionales. Diário Oficial 1994; 24 jun.

4. Congreso de Colombia. Ley no 1.562. Por la cual se modifica el Sistema de Riesgos Laborales y se dictan otras disposiciones en materia de salud ocupacional. Diário Oficial 2012; 11 jul.

5. Ministerio de Salud y Protección Social. Plan Decenal Salud Pública 2012-2021. Bogotá: Ministerio de Salud y Protección Social; 2012.

6. Ministerio del Trabajo; Organización Iberoamericana de Seguridad Social. Plan Nacional de Seguridad y Salud en el Trabajo. Bogotá: Ministerio del Trabajo; 2014.

7. Arango Soler JM, Luna García JE, CorreaMoreno YA, Campos AC. Marco legal de los riesgos profesionales y la salud ocupacional en Colombia, siglo xx. Rev Salud Pública 2013; 15:354-65.

8. Ministerio de Trabajo y Seguridad Social. Resolución no 2.400 , de 1979 . Por la cual se establecen algunas disposiciones sobre vivienda, higiene y seguridad en los establecimientos de trabajo. Diário Oficial 1979; 22 may.

9. Ministerio de Trabajo y Seguridad Social. Resolución no 2.013, de 1986. Por la cual se reglamenta la organización y funcionamiento de los Comites de Medicina, Higiene Y Seguridad Industrial en los lugares de trabajo (actualmente Comité Paritario de Salud Ocupacional). Diário Oficial 1986; 8 jun.
10. Ministerio de Trabajo y Seguridad Social. Resolución Conjunta no 1.016, de 1989. Por la cual se reglamenta la organización, funcionamiento $\mathrm{y}$ forma de los programas de salud ocupacional que deben desarrollar los patronos o empleadores en el país. Diário Oficial 1989; 31 mar.

11. Ministerio de Trabajo. Decreto no 1.072, de 2015. Por medio del cual se expide el Decreto Único Reglamentario del Sector Trabajo. Diário Oficial 2015; 26 may.

12. Ministerio de Trabajo. Decreto no 312, de 2019. Por lo cuál se definen los Estándares Mínimos del Sistema de Gestión de la Seguridad y Salud en el Trabajo SG-SST. Diário Oficial 2019; 13 feb.

13. Ministerio de Comercio Industria y Turismo. Decreto no 957. Por el cual se adiciona el capítulo 13 al Título 1 de la Parte 2 del Libro 2 del Decreto no 1.074 de 2015, Decreto Único del Sector Comercio, Industria y Turismo y se reglamenta el artículo 2o de la Ley no 590 de 2000, modificado por el artículo 43 de la Ley no 1.450 de 2011. Diário Oficial 2019; 13 jun.

14. Ortega Ruiz CA. Inclusión de las TIC en la empresa colombiana. Suma Negocios 2014; 5:29-33.

15. García OM. Políticas diferenciales para MIPYMES. Bitácora Económica 2020; 8:20-4.

16. Ministerio del Trabajo. 656 mil Mipymes y sector agropecuario se beneficiarán con nuevas exigencias del Sistema de Seguridad y Salud en el Trabajo. https://www.mintrabajo.gov. co/web/guest/prensa/comunicados/2019/ febrero/656-mil-mipymes-y-sector-agrope cuario-se-beneficiaran-con-nuevas-exigen cias-del-sistema-de-seguridad-y-salud-en-eltrabajo (accedido el 11/Abr/2020).

17. Ministerio del Trabajo. Informe ejecutivo: II Encuesta Nacional de Condiciones de Seguridad y Salud en el Trabajo en el Sistema General de Riesgos. Bogotá: Ministerio del Trabajo; 2013. 
18. Barreiro OLF, González JST, Betancur NAP. Diagnóstico de las necesidades básicas de las empresas del sector del Valle de Aburrá (Envigado, Sabaneta, Itagüí, La Estrella, Caldas). Sabaneta: Facultad de Negocios y Ciencias Empresariales, Corporación Universitaria de Sabaneta; 2014.

19. Federación de Aseguradores Colombianos. Estadísticas de accidente y enfermedad laboral. https://sistemas.fasecolda.com/rldatos/ (accedido el 10/May/2020)

20. Vega-Monsalve NDC. Nivel de implementación del Programa de Seguridad y Salud en el Trabajo en empresas de Colombia del territorio Antioqueño. Cad Saúde Pública 2017; 33:e00062516.

21. Instituto Colombiano de Normas Técnicas y Certificación. Guía para la identificación de los peligros y la valoración de los riesgos en seguridad y salud ocupacional: GTC 45. En: Instituto Colombiano de Normas Técnicas y Certificación, editor. Compendio: normas fundamentales sobre gestión de la calidad y documentos de orientación para su aplicación. 4a Ed. Bogotá: Instituto Colombiano de Normas Técnicas y Certificación; 2012. p. 1-38.
22. Briceño L. Prevención de riesgos ocupacionales en empresas colombianas. Rev Cienc Salud (Bogotá) 2003; 1:31-44.

23. Yoon SJ, Lin HK, Chen G, Yi S, Choi J, Rui Z. Effect of occupational health and safety management system on work-related accident rate and differences of occupational health and safety management system awareness between managers in South Korea's construction industry. Saf Health Work 2013; 4:201-9.

24. Ramos D, Afonso P, Rodrigues MA. Integrated management systems as a key facilitator of occupational health and safety risk management: a case study in a medium sized waste management firm. J Clean Prod 2020; 262:121346.

25. La mipymes impulsan la generación de empleo en Colombia. Semana 2016; 16 mar. https:// www.semana.com/edicion-impresa/pais/arti culo/la-mipymes-colombianas-contribuyencon-cifras-de-empleo-2016/221479/. 


\section{Abstract}

This study aimed to describe the scenario of workplace safety and health (WSH) based on the component of the Occupational Safety and Health Management System (SGSST), the identification and control of occupational hazards, and the Colombian legislation on WSH for micro businesses, focusing on the commercial and services sectors in the Los Naranjos neighborhood in Itagüi, Antioquia, Colombia. The study followed a descriptive design with voluntary participation by 50 micro businesses. A three-phase questionnaire was used: theoretical review, expert validation, and pilot study. The questionnaire included the company's characteristics, components of the SGSST, WSH legislation, and hazard management, which included the identification and control of hazards. $88 \%$ of the micro businesses do not assign a specific person to manage the SGSST, and 72\% do not permanently enroll their workers in the Comprehensive General Social Security System. Physical and chemical hazards are not identified and controlled consistently in $34 \%$ and $38 \%$ of the micro businesses, respectively. On average, $93.86 \%$ of the micro businesses are not aware of the Colombian legislation on WSH. The WSH scenario in a small sector of commercial and services businesses is not encouraging in terms of the SGSST, hazard management, and adequacy and implementation of Colombian laws on WSH, which are mandatory for workers' care.

Occupational Health; Occupational Risks; Risk Management; OHSAS 18000

\section{Resumo}

Esta pesquisa tinha como objetivo descrever o cenário da segurança e da saúde no trabalho (SST) com base nos componentes do Sistema de Gerenciamento de Segurança e Saúde no Trabalho (SGSST), a identificação e o controle de riscos ocupacionais e as normas colombianas relacionadas com a SST de microempresas dos setores de comércio e serviços do bairro de Los Naranjos, no Município de Itagüí, Antioquia, Colômbia. A pesquisa seguiu um padrão descritivo, com a participação voluntária de 50 microempresas. Foi utilizado um questionário elaborado para a pesquisa em três fases: revisão teórica, validação por especialistas e aplicação. Constavam do questionário: características da empresa, componentes do SGSST, normas de SST e gerenciamento de riscos, incluindo a sua identificação e controle. Os resultados indicam que 88\% das microempresas não designam uma pessoa específica para lidar com a SGSST, e 72\% não vinculam de forma permanente os seus funcionários ao Sistema Geral de Seguridade Social Integral. Os riscos físicos e químicos não são identificados nem controlados de maneira constante em $34 \%$ e $38 \%$ das microempresas, respectivamente. Noventa e três vírgula oitenta e seis porcento das microempresas não conhecem as normas colombianas relacionadas com a SST. O cenário da SST em uma pequena amostra do setor de comércio e serviços não é alentador no que diz respeito à adequação ao SGSST, ao gerenciamento de riscos e à adequação e implementação das normas colombianas relacionadas com a SST, compulsórias para a proteção dos trabalhadores.

Saúde do Trabalhador; Riscos Ocupacionais;

Gestão de Riscos; OHSAS 18000
Recibido el 18/Jun/2020

Versión final presentada el 03/May/2021

Aprobado el 07/May/2021 